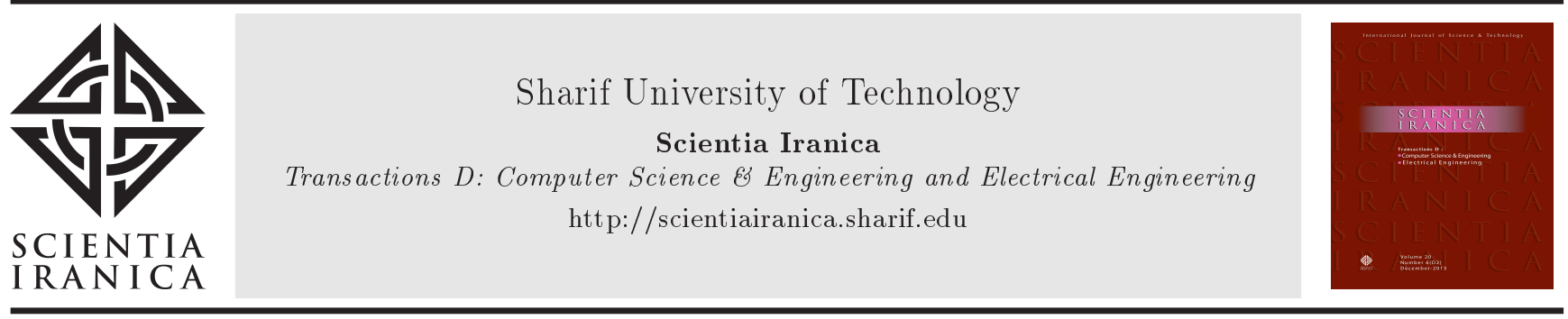

\title{
Two sufficient conditions for the existence of path factors in graphs
}

\author{
S. Zhou ${ }^{\mathrm{a}, *}$, F. Yang ${ }^{\mathrm{a}}$, and L. Xu ${ }^{\mathrm{b}}$ \\ a. School of Science, Jiangsu University of Science and Technology, Mengxi Road 2, Zhenjiang, Jiangsu 212003, P. R. China. \\ b. Department of Mathematics, Changji University, Changji, Xinjiang 831100, P. R. China.
}

Received 9 September 2017; received in revised form 20 June 2018; accepted 15 September 2018

\author{
KEYWORDS \\ Network; \\ $P_{\geq n}$-factor; \\ $\left(P_{\geq n}, m\right)$-factor \\ deleted graph; \\ $\left(P_{\geq n}, k\right)$-factor critical \\ graph; \\ Toughness; \\ Connectivity.
}

\begin{abstract}
A graph $G$ is called a $\left(P_{\geq n}, k\right)$-factor critical graph if $G-U$ has a $P_{\geq_{n}}$-factor for any $U \subseteq V(G)$ with $|U|=k$. A graph $G$ is called a $\left(P_{\geq n}, m\right)$-factor deleted graph if $G-E^{\prime}$ contains a $P_{\geq n}$-factor for any $E^{\prime} \subseteq E(G)$ with $\left|E^{\prime}\right|=m$. In this paper, we obtain two results for graphs to be $\left(P_{\geq_{n}}, k\right)$-factor critical graphs or $\left(P_{\geq n}, m\right)$-factor deleted graphs. The two results are best possible in some conditions.
\end{abstract}

(C) 2019 Sharif University of Technology. All rights reserved.

\section{Introduction}

Many real-world networks can conveniently be modelled by graphs or networks. Examples include a railroad network with nodes presenting railroad stations, and links corresponding to railways between two stations, a communication network with nodes and links modelling cities and communication channels, respectively, or the World Wide Web with nodes presenting Web pages, and links corresponding to hyperlinks between them. In our daily life, many problems in network design and optimization, e.g., building blocks, the file transfer problems in computer networks, coding design, scheduling problems and so on, are related to the factors and factorizations of graphs $[1,2]$. For example, file transfer problem in computer networks can be converted into factorizations of graphs. The problem on telephone network design

\footnotetext{
*. Corresponding author. Tel.: +86 13805287659; Fax: +8651184401171

E-mail address: zsz_cumt@163.com (S. Zhou)
}

can be converted into $P_{2}$-factors of graphs. Many other applications in this field can be found in a current survey [1]. It is well known that a graph can represent a network. Vertices and edges of the graph model nodes and links between the nodes in the network. Henceforth, we use the term graph instead of network.

In this paper, we deal with only finite and undirected graphs without loops or multiple edges. Let $G$ be a graph. We denote by $V(G)$ the vertex set of $G$ and by $E(G)$ the edge set of $G$. Given $S \subseteq V(G)$, let $G[S]$ denote the subgraph of $G$ induced by $S$, and set $G-S=G[V(G) \backslash S]$. Especially, we write:

$$
G-x=G-\{x\} \text { if } S=\{x\}
$$

Given a vertex $x$ of $G$, let $N_{G}(x)$ denote the set of vertices adjacent to $x$ in $G$ and $d_{G}(x)=\left|N_{G}(x)\right|$ denote the degree of $x$ in $G$. Given $E^{\prime} \subseteq E(G)$, let $G-E^{\prime}$ denote the subgraph obtained from $G$ by deleting $E^{\prime}$. In particular, we write $G-e=G-\{e\}$ if $E^{\prime}=\{e\}$. Let $\kappa(G)$ denote the connectivity of $G$ and $\lambda(G)$ denote the edge-connectivity of $G$. Chvátal [3] presented the parameter of toughness of a graph $G$, denoted by $t(G)$, 
defined as follows:

$$
\begin{gathered}
t(G)=\min \left\{\frac{|X|}{\omega(G-X)}: X \subseteq V(G),\right. \\
\omega(G-X) \geq 2\}
\end{gathered}
$$

if $G$ is not a complete graph, where $\omega(G-X)$ denotes the number of connected components in $G-X$. Otherwise, $t\left(K_{n}\right)=+\infty$, where $K_{n}$ denotes a complete graph of order $n$. We refer the reader to [4] for the notation and terminology which are used but not described here.

A path factor of a graph $G$ is a spanning subgraph of $G$ with each component being a path. We denote by $P_{n}$ the path on $n$ vertices. A $P_{n}$-factor of $G$ is defined as a spanning subgraph of $G$ with each component isomorphic to $P_{n}$. A path factor with each component having at least $n$ vertices is called a $P_{\geq n}$-factor. It is obvious that a path factor is a generalization of a perfect matching, which is a $P_{2}$-factor. Path factors in graphs have attracted a great deal of attention. Wang [5] characterized the bipartite graphs with $P_{\geq 3^{-}}$ factors. Kaneko [6] generalized this theorem to general graphs. For other results on graph factors, see [7-21].

A graph $G$ is called a $\left(P_{\geq n}, k\right)$-factor critical graph if $G-U$ has a $P_{\geq_{n}}$-factor for any $U \subseteq V(G)$ with $|U|=k$. It is obvious that a $\left(P_{\geq n}, 0\right)$-factor critical graph admits a $P_{\geq n}$-factor. A graph $G$ is called a $\left(P_{\geq n}, m\right)$-factor deleted graph if $G-E^{\prime}$ contains a $P_{\geq n^{-}}$ factor for any $E^{\prime} \subseteq E(G)$ with $\left|E^{\prime}\right|=m$. Clearly, a $\left(P_{\geq n}, 0\right)$-factor deleted graph has a $P_{\geq n}$-factor. A $\left(P_{\geq n}, m\right)$-factor deleted graph is simply called a $P_{\geq n^{-}}$ factor deleted graph if $m=1$.

A graph $R$ is called a factor-critical graph if $R-x$ has a perfect matching for every vertex $x$ in $R$. A graph $H$ is called a sun if $H=K_{1}, H=K_{2}$ or $H$ is the corona of a factor-critical graph, $R$, having at least 3 vertices, i.e., $H$ is obtained from $R$ by adding a new vertex $w=w(v)$ together with a new edge, $v w$, for every $v \in V(R)$. A big sun is a sun having at least 6 vertices. A component of $G$ is called a sun component if it is isomorphic to a sun. The number of sun components of $G$ is denoted by $\operatorname{sun}(G)$.

The following result for $P_{\geq 3}$-factor was first obtained by Kaneko [6]. Kano et al. [22] gave a new proof of this theorem.

Theorem 1 [6]. A graph $G$ contains a $P_{\geq 3}$-factor if and only if $\operatorname{sun}(G-S) \leq 2|S|$ for every $S \subseteq V(G)$.

A claw is a graph isomorphic to $K_{1,3}$. A graph is said to be a claw-free graph if it does not contain induced claw. For a 2-connected claw-free graph $G$, Kelmans [23] showed a sufficient condition for the existence of $P_{3}$-factors in $G-\{x\}$ for any $x \in V(G)$, and presented a sufficient condition for the existence of $P_{3}$-factors in $G-\{e\}$ for any $e \in E(G)$. Naturally, motivated by the two results, we consider two more general problems:

1. Does $G-U$ have a $P_{\geq 3}$-factor for any $U \subseteq V(G)$ with $|U|=k$, or is a graph $G$ a $\left(P_{\geq 3}, k\right)$-factor critical graph?

2. Does $G-E^{\prime}$ have a $P_{\geq 3}$-factor for any $E^{\prime} \subseteq E(G)$ with $\left|E^{\prime}\right|=m$, or is a graph $G$ a $\left(P_{\geq 3}, m\right)$-factor deleted graph?

In this paper, we investigate the relationship between toughness and $\left(P_{>3}, m\right)$-factor deleted graphs and $\left(P_{\geq 3}, k\right)$-factor critical graphs, and obtain two toughness conditions for the existence of $\left(P_{\geq 3}, m\right)$ factor deleted graphs and $\left(P_{\geq 3}, k\right)$-factor critical graphs. Our main results are the following theorems, which imply that the two problems above are true.

Theorem 2. Let $m$ be an integer with $m \geq 1$, and let $G$ be a graph with $\kappa(G) \geq \frac{3 m+1}{2}$. If $t(G)>\frac{3}{4}-\frac{1}{2(m+1)}$, then $G$ is a $\left(P_{\geq 3}, m\right)$-factor deleted graph.

Remark 1. The result in Theorem 2 is sharp. In order to show this, we construct a graph $G=K_{\frac{3 m+1}{2}} \vee$ $2(m+1) K_{2}$, where $m \geq 1$ is an odd integer and $\vee$ means "join". It is obvious that $t(G)=\frac{\frac{3 m+1}{2}}{2(m+1)}=\frac{3 m+1}{4(m+1)}=$ $\frac{3}{4}-\frac{1}{2(m+1)}$. Set $E^{\prime} \subseteq E\left(2(m+1) K_{2}\right)$ with $\left|E^{\prime}\right|=m$ and $G^{\prime}=G-E^{\prime}$. We choose $S=V\left(K_{\frac{3 m+1}{2}}\right) \subseteq V\left(G^{\prime}\right)$, and so $|S|=\frac{3 m+1}{2}$. Thus, we have:

$$
\begin{aligned}
\operatorname{sun}\left(G^{\prime}-S\right) & =(m+2)+2 m \\
& =3 m+2>3 m+1=2|S| .
\end{aligned}
$$

In terms of Theorem $1, G^{\prime}$ has no $P_{\geq 3}$-factor. Combining this with the definition of $\left(P_{\geq 3}, m\right)$-factor deleted graph, $G$ is not a $\left(P_{\geq 3}, m\right)$-factor deleted graph.

Theorem 3. Let $k \geq 0$ be an integer. A graph $G$ with $\kappa(G) \geq k+2$ is a $\left(P_{\geq 3}, k\right)$-factor critical graph if $t(G) \geq \frac{k+1}{2}$.

Remark 2. Let $k \geq 0$ be an integer. We now show that the conditions $t(G) \geq \frac{k+1}{2}$ and $\kappa(G) \geq k+2$ in Theorem 3 cannot be replaced by $t(G) \geq \frac{k+1}{3}$ and $\kappa(G) \geq k+1$. We consider the graph $G=K_{k+1} \vee 3 K_{2}$, and choose $U \subseteq V\left(K_{k+1}\right)$ with $|U|=k$. Obviously, $t(G)=\frac{k+1}{3}$ and $\kappa(G)=k+1$. Let $G^{\prime}=G-U$. For $S=V\left(K_{k+1}\right) \backslash U$, we have:

$$
\operatorname{sun}\left(G^{\prime}-S\right)=3>2=2|S| \text {. }
$$

In view of Theorem $1, G^{\prime}$ has no $P_{\geq 3}$-factor; thus, $G$ is not a $\left(P_{\geq 3}, k\right)$-factor critical graph. 


\section{Proof of Theorem 2}

If $G$ is a complete graph, then it is obvious that $G$ is a $\left(P_{\geq 3}, m\right)$-factor deleted graph by $\lambda(G) \geq \kappa(G) \geq \frac{3 m+1}{2}$ and the definition of $\left(P_{\geq 3}, m\right)$-factor deleted graph. Hence, we may assume that $G$ is a non-complete graph.

For any $E^{\prime} \subseteq E(G)$ with $\left|E^{\prime}\right|=m$, we write $G^{\prime}=$ $G-E^{\prime}$. It is obvious that $V\left(G^{\prime}\right)=V(G)$ and $E\left(G^{\prime}\right)=$ $E(G) \backslash E^{\prime}$. To prove the theorem, we only need to verify that $G^{\prime}$ admits a $P_{\geq 3}$-factor. By contradiction, we assume that $G^{\prime}$ has no $P_{\geq 3}$-factor. Then it follows from Theorem 1 that there exists some subset $S \subseteq$ $V\left(G^{\prime}\right)$ satisfying:

$$
\operatorname{sun}\left(G^{\prime}-S\right)>2|S| \text {. }
$$

It follows from $\lambda(G) \geq \kappa(G) \geq \frac{3 m+1}{2}$ that $G^{\prime}=$ $G-E^{\prime}$ is connected; therefore:

$$
\operatorname{sun}\left(G^{\prime}\right) \leq \omega\left(G^{\prime}\right)=1 .
$$

Claim 1. $S \neq \emptyset$.

Proof. If $S=\emptyset$, then by Eqs. (1) and (2) we obtain:

$$
1=\omega\left(G^{\prime}\right) \geq \operatorname{sun}\left(G^{\prime}\right) \geq 1,
$$

that is:

$$
\operatorname{sun}\left(G^{\prime}\right)=\omega\left(G^{\prime}\right)=1 .
$$

Since $m \geq 1$ is an integer, we have:

$$
\lambda(G) \geq \kappa(G) \geq \frac{3 m+1}{2} \geq 2,
$$

which implies:

$$
\left|V\left(G^{\prime}\right)\right|=|V(G)| \geq 3,
$$

and:

$$
\begin{aligned}
\lambda\left(G^{\prime}\right) & =\lambda\left(G-E^{\prime}\right) \geq \lambda(G)-\left|E^{\prime}\right| \geq \frac{3 m+1}{2}-m \\
& =\frac{m+1}{2} .
\end{aligned}
$$

If $m=1$, then $G^{\prime}=G-E^{\prime}$ is a big sun by Eq. (3), Eq. (4), and the definition of a big sun. Let $R$ denote the factor-critical graph in $G^{\prime}=G-E^{\prime}$. It is obvious that there exists $x \in V(R)$ with $\omega(G-\{x\})=2$. Thus, we obtain:

$$
\frac{1}{2}=\frac{3}{4}-\frac{1}{2(m+1)}<t(G) \leq \frac{|\{x\}|}{\omega(G-\{x\})}=\frac{1}{2},
$$

for $m=1$, which is a contradiction.

If $m \geq 2$, then from Eq. (5) we have:

$$
\lambda\left(G^{\prime}\right) \geq \frac{m+1}{2}>1 \text {. }
$$

In view of the integrity of $\lambda\left(G^{\prime}\right)$, we obtain $\lambda\left(G^{\prime}\right) \geq 2$, and so $\operatorname{sun}\left(G^{\prime}\right)=0$, which contradicts Eq. (3). Claim 1 is proved. $\square$

In the following, we shall consider two cases.
Case 1. $S$ is not a vertex cut set of $G$.

Since $\kappa(G) \geq \frac{3 m+1}{2}, G$ is a connected graph. Thus, we have $\omega(G-S)=\omega(G)=1$. After deleting an edge in a graph, the number of its components increases by at most 1 . Hence, we have:

$$
\begin{aligned}
\operatorname{sun}\left(G^{\prime}-S\right) & \leq \omega\left(G^{\prime}-S\right)=\omega\left(G-S-E^{\prime}\right) \\
& \leq \omega(G-S)+m=m+1 .
\end{aligned}
$$

According to Eqs. (1) and (6), we obtain:

$$
2|S|<\operatorname{sun}\left(G^{\prime}-S\right) \leq m+1
$$

that is:

$$
|S|<\frac{m+1}{2} .
$$

It follows from Eq. (7), $\lambda(G-S) \geq \kappa(G-S)$ and $\kappa(G) \geq \frac{3 m+1}{2}$ that:

$$
\begin{aligned}
\lambda(G-S) & \geq \kappa(G-S) \geq \kappa(G)-|S| \\
& >\frac{3 m+1}{2}-\frac{m+1}{2}=m .
\end{aligned}
$$

In terms of the integrity of $\lambda(G-S)$, we have:

$$
\lambda(G-S) \geq m+1 .
$$

Using Eq. (8), we obtain:

$$
\lambda\left(G^{\prime}-S\right) \geq \lambda(G-S)-m \geq 1,
$$

and so:

$$
\operatorname{sun}\left(G^{\prime}-S\right) \leq \omega\left(G^{\prime}-S\right)=1,
$$

which contradicts Eq. (1) by Claim 1.

Case 2. $S$ is a vertex cut set of $G$.

Since $S$ is a vertex cut set of $G$ and $\kappa(G) \geq \frac{3 m+1}{2}$, we obtain:

$$
\omega(G-S) \geq 2,
$$

and:

$$
|S| \geq \frac{3 m+1}{2} .
$$

Note that $\operatorname{sun}\left(G^{\prime}-S\right) \leq \omega\left(G^{\prime}-S\right) \leq \omega(G-S)+$ $m$. Combining this with Eq. (1), we have:

$$
2|S|+1 \leq \operatorname{sun}\left(G^{\prime}-S\right) \leq \omega(G-S)+m,
$$

that is:

$$
\omega(G-S) \geq 2|S|-m+1 .
$$


In view of Eqs. (9) and (11), $t(G)>\frac{3}{4}-\frac{1}{2(m+1)}$, and the definition of $t(G)$, we obtain:

$$
\frac{3}{4}-\frac{1}{2(m+1)}<t(G) \leq \frac{|S|}{\omega(G-S)} \leq \frac{|S|}{2|S|-m+1},
$$

which implies:

$$
2(m-1)|S|<(m-1)(3 m+1) .
$$

If $m=1$, then by Eq. (12) we have $0<0$, which is impossible. If $m \geq 2$, then it follows from Eq. (12) that:

$$
|S|<\frac{3 m+1}{2}
$$

which contradicts Eq. (10). Theorem 2 is proved. $\square$

\section{Proof of Theorem 3}

It is obvious that a complete graph $G$ with $\kappa(G) \geq$ $k+2$ is a $\left(P_{\geq 3}, k\right)$-factor critical graph. Hence, we may assume that $G$ is not a complete graph.

For any $U \subseteq V(G)$ with $|U|=k$, we write $G^{\prime}=$ $G-U$. To verify the theorem, we only need to prove that $G^{\prime}$ has a $P_{\geq 3}$-factor. On the contrary, we assume that $G^{\prime}$ has no $P_{\geq 3}$-factor. Then, there exists some subset $S \subseteq V\left(G^{\prime}\right)$ such that:

$$
\operatorname{sun}\left(G^{\prime}-S\right) \geq 2|S|+1,
$$

by Theorem 1 .

If $S=\emptyset$, then we have:

$$
\operatorname{sun}\left(G^{\prime}\right)=\operatorname{sun}(G-U)=0,
$$

by $|U|=k$ and $\kappa(G) \geq k+2$, which contradicts Eq. (13). Hence, we may assume that $S \neq \emptyset$. In the following, we consider two cases by the value of $\operatorname{sun}\left(G^{\prime}-S\right)$.

Case 1. $\operatorname{sun}\left(G^{\prime}-S\right) \leq 1$.

It follows from $S \neq \emptyset$ and Eq. (13) that:

$$
\operatorname{sun}\left(G^{\prime}-S\right) \geq 2|S|+1 \geq 3,
$$

which contradicts $\operatorname{sun}\left(G^{\prime}-S\right) \leq 1$.

Case 2. $\operatorname{sun}\left(G^{\prime}-S\right) \geq 2$.

Note that $\omega(G-(S \cup U))=\omega\left(G^{\prime}-S\right) \geq \operatorname{sun}\left(G^{\prime}-\right.$

$S) \geq 2$. Combining this with $t(G) \geq \frac{k+1}{2}$ and the definition of $t(G)$, we obtain:

$$
\begin{aligned}
\frac{k+1}{2} \leq & t(G) \leq \frac{|S \cup U|}{\omega(G-(S \cup U))} \leq \frac{|S|+k}{\operatorname{sun}\left(G^{\prime}-S\right)} \\
= & \frac{|S|}{\operatorname{sun}\left(G^{\prime}-S\right)}+\frac{k}{\operatorname{sun}\left(G^{\prime}-S\right)} \leq \frac{|S|}{\operatorname{sun}\left(G^{\prime}-S\right)} \\
& +\frac{k}{2}
\end{aligned}
$$

which implies:

$$
\operatorname{sun}\left(G^{\prime}-S\right) \leq 2|S|
$$

which contradicts Eq. (13). This completes the proof of Theorem 3.

\section{Conclusion}

In this paper, we investigated the existence of $\left(P_{\geq 3}, m\right)$ factor deleted graphs and $\left(P_{\geq 3}, k\right)$-factor critical graphs and obtained two results for them, which were two extensions of the previous studies. Path factors in graphs or networks have attracted a great deal of attention due to their applications in network design, statistical mechanics, information transmission in networks, and so on. Hence, there is theoretical and practical significance in investigating the problem of path factors in graphs or networks.

\section{Acknowledgments}

The authors would like to thank the anonymous referees for their kind help and valuable suggestions, which led to an improvement of this paper. This work was sponsored by 333 Project of Jiangsu Province and Six Big Talent Peak of Jiangsu Province (Grant No. JY-022), and supported by the National Natural Science Foundation of China (Grant No. 11501256, 61503160) and the Natural Science Foundation of Xinjiang Province of China (Grant No. 2015211A003).

\section{References}

1. Alspach, B., Heinrich, K., and Liu, G. "Orthogonal factorizations of graphs", in: J.H. Dinitz, D. R. Stinson (Eds.), Contemporary Design Theory: A Collection of Surveys, Wiley, New York, pp. 13-37 (1992).

2. Rezaei, S. and Afshin Hemmatyar, A.M. "CMORC: Class-based Multipath On-demand Routing protocol for Cognitive radio networks", Scientia Iranica, 24(6), pp. 3117-3131 (2017).

3. Chvátal, V. "Tough graphs and Hamiltonian circuits", Discrete Mathematics, 5, pp. 215-228 (1973).

4. Bondy, J.A. and Murty, U.S.R., Graph Theory, Springer, Berlin (2008).

5. Wang, H. "Path factors of bipartite graphs", Journal of Graph Theory, 18, pp. 161-167 (1994).

6. Kaneko, A. "A necessary and sufficient condition for the existence of a path factor every component of which is a path of length at least two", Journal of Combinatorial Theory, Series B, 88, pp. 195-218 (2003).

7. Zhou, S. and Zhang, T. "Some existence theorems on all fractional $(g, f)$-factors with prescribed properties", Acta Mathematicae Applicatae Sinica, English Series, 34(2), pp. 344-350 (2018). 
8. Zhou, S., Yang, F., and Sun, Z. "A neighborhood condition for fractional ID-[a,b]-factor-critical graphs", Discussiones Mathematicae Graph Theory, 36(2), pp. 409-418 (2016).

9. Zhou, S., Wu, J., and Zhang, T. "The existence of $P_{3_{3}}$ factor covered graphs", Discussiones Mathematicae Graph Theory, 37(4), pp. 1055-1065 (2017).

10. Zhou, S. "Some results about component factors in graphs", RAIRO-Operations Research, DOI: $10.1051 / \mathrm{ro} / 2017045$.

11. Zhou, S., Liu, H., and Zhang, T. "Randomly orthogonal factorizations with constraints in bipartite networks", Chaos, Solitons and Fractals, 112, pp. 3135 (2018).

12. Zhou, S. and Bian, Q. "Subdigraphs with orthogonal factorizations of digraphs (II)", European Journal of Combinatorics, 36, pp. 198-205 (2014).

13. Zhou, S. "Remarks on orthogonal factorizations of digraphs", International Journal of Computer Mathematics, 91(10), pp. 2109-2117 (2014).

14. Zhou, S. "A sufficient condition for a graph to be an $(a, b, k)$-critical graph", International Journal of Computer Mathematics, 87(10), pp. 2202-2211 (2010).

15. Zhou, S. and Sun, Z. "Neighborhood conditions for fractional ID- $k$-factor-critical graphs", Acta Mathematicae Applicatae Sinica, English Series, 34(3), pp. 636-644 (2018).

16. Kawarabayashi, K., Matsuda, H., Oda, Y., and Ota, K. "Path factors in cubic graphs", Journal of Graph Theory, 39, pp. 188-193 (2002).

17. Razzazi, M. and Sepahvand, A. "Time complexity of two disjoint simple paths", Scientia Iranica, 24(3), pp. 1335-1343 (2017).

18. Johnson, M., Paulusma, D., and Wood, C. "Path factors and parallel knock-out schemes of almost clawfree graphs", Discrete Mathematics, 310, pp. 14131423 (2010).

19. Gao, W., Guirao, J., and Wu, H. "Two tight independent set conditions for fractional $(g, f, m)$-deleted graphs systems", Qualitative Theory of Dynamical Systems, 17(1), pp. 231-243 (2018).

20. Gao, W. and Wang, W. "Degree sum condition for fractional ID- $k$-factor-critical graphs", Miskolc Mathematical Notes, 18(2), pp. 751-758 (2017).

21. Gao, W., Liang, L., and Chen, Y. "An isolated toughness condition for graphs to be fractional $(k, m)$ deleted graphs", Utilitas Mathematica, 105, pp. 303316 (2017).

22. Kano, M., Katona, G.Y., and Király, Z. "Packing paths of length at least two", Discrete Mathematics, 283, pp. 129-135 (2004).

23. Kelmans, A. "Packing 3-vertex paths in claw-free graphs and related topics", Discrete Applied Mathematics, 159, pp. 112-127 (2011).

\section{Biographies}

Sizhong Zhou was born in Anhui Province, China. He received his BS and MS degrees from China University of Mining and Technology. Since 2003, he has been with the School of Science at the Jiangsu University of Science and Technology, where he was appointed as an Associate Professor of Mathematics in 2009. His current research interests focus on graph theory.

Fan Yang was born in Jiangxi Province, China. She received her BS degree from Jiangxi Normal University, and her MS and PhD degrees from Central China Normal University. Since 2011, she has been with the School of Science at the Jiangsu University of Science and Technology, where she was appointed as an Associate Professor of Mathematics in 2015. Her current research interests focus on graph theory.

Lan Xu was born in Jiangsu Province, China. She is a Professor in the Department of Mathematics at Changji University. Her current research interests focus on algebra and graph theory. 